\title{
ORIGINAL ARTICLE Blocking ETV6/RUNX1-induced MDM2 overexpression by Nutlin-3 reactivates p53 signaling in childhood leukemia
}

\author{
U Kaindl ${ }^{1,3}$, M Morak $^{1,3}$, C Portsmouth ${ }^{1}$, A Mecklenbräuker ${ }^{1}$, M Kauer $^{1}$, M Zeginigg ${ }^{1}$, A Attarbaschi ${ }^{2}$, OA Haas $^{1,2}$ \\ and R Panzer-Grümayer ${ }^{1}$
}

\begin{abstract}
ETV6/RUNX1 $(E / R)$ is the most common fusion gene in childhood acute lymphoblastic leukemia. It is responsible for the initiation of leukemia but also indispensable for disease maintenance and propagation, although its function in these latter processes is less clear. We therefore investigated the effects of the perceived p53 pathway alterations in model cell lines and primary leukemias and, in particular, how E/R upregulates MDM2, the predominant negative regulator of $\mathrm{p} 53$. We found that E/R transactivates MDM2 in both $\mathrm{p} 53^{+/+}$and $\mathrm{p} 53^{-1-}$ HCT116 cells by binding to promoter-inherent RUNX1 motifs, which indicates that this activation occurs in a direct and $\mathrm{p} 53$-independent manner. Treatment of $E / R$-positive leukemic cell lines with Nutlin-3, a small molecule that inhibits the MDM2/p53 interaction, arrests their cell cycle and induces apoptosis. These phenomena concur with a p53-induced expression of p21, pro-apoptotic BAX and PUMA, as well as caspase 3 activation and poly ADP-ribose polymerase cleavage. The addition of DNA-damaging and p53-activating chemotherapeutic drugs intensifies apoptosis. Moreover, Nutlin-3 exposure leads to an analogous p53 accumulation and apoptotic surge in E/R-positive primary leukemic cells. Our findings clarify the role of p53 signaling in $E / R$-positive leukemias and outline the potential basis for its therapeutic exploitation in this setting.
\end{abstract}

Leukemia (2014) 28, 600-608; doi:10.1038/leu.2013.345

Keywords: ETV6/RUNX1-positive leukemia; MDM2; p53 pathway; Nutlin-3; ETV6/RUNX1 targets

\section{INTRODUCTION}

With an incidence of approximately $25 \%$ ETV6/RUNX1 (E/R) is the most frequent fusion gene in childhood B-cell precursor leukemia. ${ }^{1}$ The encoded chimeric protein retains the $\mathrm{N}$-terminal region of ETV6 and almost the entire RUNX1 protein including the DNA-binding domain. ${ }^{2}$ Despite the lack of high-risk features, up to $20 \%$ of affected children that are treated according to Berlin-FrankfurtMünster-based protocols experience predominantly late relapses. ${ }^{3}$ In contrast to commonly expressed opinions, these relapses are often associated with drug resistance and poor outcome. ${ }^{1,4,5}$

There is ample evidence that the $E / R$ gene fusion is most likely the initiating event in leukemia development. Although it provides the affected cells with an essential survival advantage, they nevertheless require additional mutations to evolve into overt leukemia. ${ }^{2,6-10}$ Among the many possible mechanisms through which E/R fosters the survival of affected cells is the upregulation of survivin, which was recently identified by a small interfering RNAmediated fusion gene knock-down (KD)., ${ }^{6,7}$ To better understand the transcriptional regulation and regulatory programs that $E / R$ imposes on the leukemic cells, we also generated a lentiviral short hairpin RNA-carrying system to KD the chimeric protein in E/R-harboring leukemic cell lines. ${ }^{112}$ These experiments facilitated the identification of the MDM2 gene, the predominant negative regulator of p53 as a distinctively regulated gene. We therefore considered its upregulation of special relevance to the survival of these fusion gene-harboring leukemias.

TP53 has the central role in the regulation of cell cycle, apoptosis, DNA repair and senescence and thereby acts as a gatekeeper of genomic integrity. It is activated by various stress signals and, in response, turns off proliferation by arresting the cell cycle to enable the appropriate repair of damaged DNA. ${ }^{13,14}$ In case this fails, p53 also triggers apoptosis and senescence. p53 is precisely controlled by MDM2, which is an E3 ubiquitin ligase that targets p53 for ubiquitin-dependent degradation and therefore functions as a crucial negative regulator. In a feedback loop, p53 activates MDM2, which in turn inactivates p53 to prevent prolonged activation of $\mathrm{p} 53$. Thus, under physiological conditions these two proteins regulate each other in a dynamic manner and any imbalances result in a functional disturbance whose outcome heavily depends on the type and state of the affected cell. ${ }^{14}$

In consideration of its critical role as tumor suppressor, it is not surprising that TP53 is mutated in approximately $50 \%$ of all cancers, and functionally silenced in many others. ${ }^{15}$ However, with an overall frequency below $5 \%$ at diagnosis and only up to $12 \%$ at relapse, TP53 mutations are uncommon in acute lymphoblastic leukemia (ALL) and particularly scarce in childhood cases. ${ }^{16,17}$ These rates also apply to the E/R-positive subgroup. ${ }^{16}$ So far, the possibility that this pathway can also be impaired by pure regulatory mechanisms, which do not involve or require mutational TP53 alterations, has not yet been addressed systematically for this particular entity.

Our recent observations are of interest in this context, as they provided the first clues that this might indeed be the case. They indicated that an E/R-induced MDM2 overexpression is the central and essential silencing factor of the p53 pathway..$^{12}$ In this study, we therefore investigated how the presence of an $E / R$ fusion gene might cause MDM2 overexpression and how it impedes p53 signaling.

${ }^{1}$ St Anna Kinderkrebsforschung, Children's Cancer Research Institute, Vienna, Austria and ${ }^{2}$ St Anna Kinderspital, Medical University Vienna, Vienna, Austria. Correspondence: Professor R Panzer-Grümayer, Leukemia Biology Group, Children's Cancer Research Institute, Zimmermannplatz 10, Vienna 1090, Austria.

E-mail: renate.panzer@ccri.at

${ }^{3}$ These authors contributed equally to this work.

Received 4 September 2013; revised 3 October 2013; accepted 7 October 2013; accepted article preview online 18 November 2013 ; advance online publication, 13 December 2013 


\section{MATERIALS AND METHODS}

\section{Cell culture}

JD Rowley (University of Chicago, IL, USA) kindly provided the E/R-positive B-cell precursor ALL cell lines AT-2 (ref. 18) and UoCB6 (ref. 19). The third $E / R$-positive leukemic cell line REH was obtained from the DSMZ (Braunschweig, Germany). All three cell lines lack genetic alterations of TP53 and MDM2 but harbor homozygous CDKN2A deletions as confirmed by single-nucleotide polymorphism arrays and fluorescence in situ hybridization. HCT116 $\mathrm{p} 53^{+/+}$ and $\mathrm{p} 53^{-/-}$cell lines were kindly provided by B Vogelstein (Johns Hopkins University, Baltimore, MD, USA). Mouse putative pro-B E/R-expressing $\mathrm{Ba} / \mathrm{F} 3$ clones and empty vector controls were established and cultured, as described previously., 60

Primary leukemic cells were obtained from bone marrow aspirations of children with ALL that were enrolled in the Austrian ALL-Berlin-Frankfurt-Münster 2000 protocol. The ethical committees of the Children's Cancer Research Institute and the St Anna Kinderspital approved this study. In accordance with the Declaration of Helsinki, we obtained written informed consent from the patients' parents for using spare material in this study.

\section{Quantitative reverse transcription-PCR}

Total RNA was extracted using TRI Reagent (MRC Inc., Cincinnati, $\mathrm{OH}$, USA) and used for complementary DNA synthesis as described previously. ${ }^{11}$ Human MDM2 transcripts, $A B L$ and GUS as endogenous controls, were quantified by TaqMan qRT-PCR using published primer probe combinations. ${ }^{12}$

\section{Cell cycle, viability and apoptosis assays}

The cell cycle distribution was assessed with the Cycletest Plus DNA Reagent Kit (Becton Dickinson, Franklin Lakes, NJ, USA) according to the manufacturer's recommendations. Cell viability was determined by 3-(4,5-dimethylthiazol-2-yl)-2,5-diphenyltetrazoliumbromid colorimetric assay (Sigma-Aldrich, St Louis, MO, USA). The proportion of apoptotic cells was determined by flow cytometry using annexin V/propidium iodide and cleaved caspase 3 stainings. All assays were performed as described previously. ${ }^{11}$

\section{Western blot analysis}

Cells were lysed, resolved and transferred as reported previously, using $60 \mu \mathrm{g}$ of total protein. ${ }^{11}$ The primary antibodies used were: anti-MYC antibody (9E10), anti-glyceraldehyde 3-phosphate dehydrogenase antibody (6C5) and anti-p53 antibody (DO-1) (Santa Cruz Biotechnology Inc., Santa Cruz, CA, USA); anti-MDM2 antibody (OP143) and anti-p21 antibody (OP64) (Calbiochem, San Diego, CA, USA); anti-BAX (\#2774) and anti-PUMA antibodies (\#4976) (Cell Signaling Technology Inc., Danvers, MA, USA); antiV5-horseradish peroxidase antibody (Invitrogen, Carlsbad, CA, USA) and anti-poly ADP-ribose polymerase antibody (C210) (Becton Dickinson). Secondary antibodies were horseradish peroxidase- or infrared dye-labeled (Bio-Rad, Hercules, CA, USA and LI-COR Biosciences, Lincoln, NE, USA, respectively) and proteins were visualized either with an enhanced chemiluminescence detection system (Thermo Scientific, Waltham, MA, USA) or membranes were scanned with the Odyssey Infrared Imaging System (LI-COR Biosciences), respectively.

\section{Quantification of intracellular proteins by flow cytometry}

For detection of p53 protein, cells were fixed and permeabilized with the FoxP3 Staining Buffer Set (Biosience, Inc., San Diego, CA, USA) according to the manufacturer's recommendation. For cleaved caspase-3 staining, cells were fixed with $2 \%$ paraformaldehyde, permeabilized with $100 \%$ ice-cold methanol and incubated with mouse anti-p53 phycoerythrin antibody and rabbit anti-active caspase-3 phycoerythrin antibody (BD Pharmingen, Franklin Lakes, $\mathrm{NJ}$, USA) for $30 \mathrm{~min}$ at room temperature. Samples were analyzed with a FACS Calibur flow cytometer (Becton Dickinson) and FlowJo software (Tree Star, Ashland, OR, USA).

\section{Luciferase reporter assays}

Luciferase assays were performed using the MDM2 reporter construct (hdm2luc01) containing a 895-bp fragment of the P2 promoter region (kindly provided by JP Blaydes, University of Southampton, UK). ${ }^{21}$ This construct was used to transiently transfect HCT116 cells together with E/R (or empty vector as control), core binding factor $\beta$ and p300 to enhance DNA binding, and pRL-TK Renilla (Promega, Madison, WI, USA) as transfection control, as described in detail elsewhere. ${ }^{6,20}$ Cells were lysed $48 \mathrm{~h}$ after transfection and luciferase activities were measured using the Dual-Luciferase Reporter Assay System (Promega).

\section{Chromatin immunoprecipitation (ChIP)}

ChIP was performed using HEK 293T, which stably expressed either $E / R$, a runt homology domain deleted $\Delta R H D-E / R$ mutant construct (kindly provided by O Williams, UK), or an empty vector, all tagged with a C-terminal V5. Two E/R-expressing HEK 293T clones were chosen (\#21 with high and \#26 with low amounts of fusion protein) to show dose dependency. In all, $2.5 \times 10^{7}$ cells were used for each immunoprecipitation sample. Cells were incubated with formaldehyde (1\%) at room temperature for $10 \mathrm{~min}$, unreacted formaldehyde was quenched by addition of glycine $(0.125 \mathrm{M}$, final concentration) and washed in cold phosphate-buffered saline with protease inhibitors (Roche, Basel, Switzerland). Nuclear extracts were prepared in $0.5 \mathrm{ml}$ ChIP lysis buffer for $15 \mathrm{~min}$ on ice. Chromatin was sonicated to an average fragment size of $500 \mathrm{bp}$ and centrifuged to remove cell debris. Fifty microliter aliquots of supernatant containing the crosslinked chromatin was transferred to fresh microfuge tubes. Two of them were supplemented with $450 \mu \mathrm{l} \mathrm{ChIP} \mathrm{dilution} \mathrm{buffer} \mathrm{and} 10 \%$ each were removed as input control; to the remaining samples the different antibodies (mouse normal IgG antibody (Santa Cruz Biotechnology Inc.) and mouse anti-V5 antibody (Life Technologies, Carlsbad, CA, USA) and the fully suspended protein A magnetic beads were added. Beads were separated on a magnetic stand and sequentially washed with various buffers (low-salt, high-salt, LiCl and TE buffers). Chromatin was eluted (elution buffer) and DNA crosslinks were reversed by adding $1 \mu \mathrm{l}$ proteinase $\mathrm{K}(20 \mathrm{mg} / \mathrm{ml})$ and incubation at $65^{\circ} \mathrm{C}$ for $2 \mathrm{~h}$. DNA was recovered using a PCR purification kit (Qiagen, Hilden, Germany) and assessed by real-time PCR. The composition of the buffers is provided in the Supplementary Information.

\section{SYBR green real-time PCR}

To amplify the putative RUNX1-binding site in the MDM2 promoter SYBR green real-time PCR was performed. The reaction included $2 \times$ Maxima SYBR Green/ROX qPCR MasterMix (Thermo Scientific), $0.4 \mu \mathrm{M}$ primers and $2 \mu \mathrm{l}$ of immunoprecipitated DNA as a template in a $25 \mu \mathrm{l}$ reaction volume. Primer sequences for the human MDM2 RUNX1 motif-positive site were: (forward) 5'-TCAAGTTCAGACACGTTCCGAA-3' and (reverse) 5'-ACTAAAGC TACAAGCAAGTCGGTG-3'. Primer sequences for the human MDM2 RUNX1-negative site were: (forward) 5'-TGATGGATATGTTTGCTG CAGG-3' and (reverse) 5'-GTGCACCAACAGACTTTAATAACTTC-3'.

\section{Bioinformatics and statistical analysis}

Bioinformatic analyses were performed in $\mathrm{R}$ statistical environment using Bioconductor packages. ${ }^{22}$ A detailed description of gene expression profiling is provided elsewhere. ${ }^{12}$ For the re-analysis of Den Boer's primary ALL data, ${ }^{23}$ CEL files were downloaded from the GEO database (GSE13425) and normalized using the gcrma algorithm. ${ }^{24}$ Detailed information on gene sets used for gene set enrichment analysis can be found in the Molecular Signatures 
Database (http://www.broad.mit.edu/gsea/msigdb/; Cambridge, USA). For all Affymetrix microarray data, probe sets with very low expression values were excluded (R package: 'panp') and one probe set with the highest variance across all samples was chosen for each gene. Differentially expressed genes were determined using a moderated $t$-test in the $\mathrm{R}$ package 'limma'. ${ }^{25}$ All $P$-values were corrected for multiple testing using the 'Benjamini-Hochberg' correction method. Differences between $E / R$-positive and $E / R$-negative samples as well as between different drug treatments were assessed by the unpaired $t$-test with Welch's correction by using the Graph Pad Prism Software (GraphPad Software, Inc., La Jolla, CA, USA). Average levels were expressed as mean \pm s.d. Statistical significance was considered when $P<0.05$.

\section{RESULTS}

E/R shuts down p53 transcription

On the basis of the previous suggestions that the $E / R$ fusion protein is primarily responsible for the deranged $p 53$ signaling, we aimed to identify differentially regulated genes by comparing microarray data of $E / R$-positive cell lines and primary leukemias with that of their fusion-negative B-cell precursor ALL counterparts. Gene set enrichment analysis revealed groups of genes that are involved in p53 signaling (Figure 1a). For additional comparisons, we also used the gene expression signature from two $E / R$-specific leukemic cell lines (REH and AT2) in which the endogenous fusion gene had been knocked down (Figure 1a). ${ }^{12}$ Two of the identified gene sets, the 'KANNAN_TP53_TARGETS_UP' and 'TP53 DOWNSTREAM TARGETS', comprise mainly activated and proposed direct targets of $\mathrm{p} 53 .^{26}$ As shown in Figure $1 \mathrm{~b}$ (upper part), $E / R \mathrm{KD}$ leads to the de-repression of p53 target genes. This pattern reverses when stably E/R-expressing $\mathrm{Ba} / \mathrm{F} 3$ cells are used as a 'first hit' model ( $P<0.05$; Figure $1 \mathrm{~b}$, bottom). ${ }^{6,20}$

We then generated a list of significantly and concordantly regulated genes from the pooled primary leukemia and KD model data set. We found that MDM2 is not only one of the most prominent members of the 50 most highly upregulated genes (Supplementary Table 1), but that its expression is significantly more pronounced in $E / R$-positive than in $E / R$-negative ALL cases (Figure 1c, left). As MDM2 overexpression only suppresses p53 signaling in human cancers with a wt TP53, we explored whether this is also the case in $E / R$-positive leukemias.

Figure 1. Deregulation of the $\mathrm{p} 53$ pathway by E/R. (a) Output from gene set enrichment analysis of primary leukemias and the $E / R K D$ model. Depicted is a summary of significantly $(P<0.05)$ de-regulated gene sets implicated in p53 signaling. (b) Heat map of the KANNAN_TP53_TARGETS_UP gene set, showing the de-repression of genes in the E/R KD model (top) and the repression of genes in $\mathrm{E} / \mathrm{R}$-expressing $\mathrm{BaF} 3$ cells (bottom). The differential expression is indicated by a color code, the respective scales are shown on top of each heat map. Positive log-ratios (log2-fold changes, log2 (FC)) are shown in red, negative log-ratios in green. (c) MDM2 expression of microarray data ${ }^{23}$ from $E / R$-positive $(\mathrm{E} / \mathrm{R}+)$ and $E / R$-negative $(\mathrm{E} / \mathrm{R}-)$ primary B-cell precursor-ALL samples (left). Quantification of MDM2 P2 transcript levels by quantitative reverse transcription-PCR (qRT-PCR). Shown is the relative expression of $M D M 2$ using $A B L$ as a standard reference for normalization in $E / R$-positive $(E / R+; n=12)$ and $E / R$-negative (E/R-; $n=11)$ primary $A L L$ samples (right). (d) qRT-PCR analysis of MDM2 transcripts in E/R-suppressed REH cells and respective controls (non-targeting short hairpin RNA). Shown are MDM2 levels determined in biological triplicates upon normalization. (e) MDM2 transcripts (top) and E/R protein levels (bottom) of two different stably E/R-expressing Ba/F3 clones (\#33 and \#48). Anti-glyceraldehyde 3-phosphate dehydrogenase was used as loading control. Bars represent the mean \pm s.d. of three independent experiments. Welch's $t$-test, ${ }^{*} P \leqslant 0.05,{ }^{*} P \leqslant 0.01$, $* * * P \leqslant 0.001$.
E/R upregulates MDM2 in a p53-independent manner and by binding to a promoter-inherent RUNX1 motif

Quantification of MDM2 transcripts in primary ALL samples confirms that the respective levels are significantly more abundant in $E / R$-positive $(n=12)$ than in $E / R$-negative $(n=11)$ cases (Figure 1c, right). In accordance, MDM2 levels were lower in REH

a

\begin{tabular}{|lr|}
\hline Gene set & P-value \\
\hline Primary leukemias & \\
\hline BIOCARTA_P53_PATHWAY & 0.009 \\
KEGG_P53_SIGNALING_PATHWAY $^{1}$ & 0.002 \\
INGA_TP53_TARGETS $^{1}$ & 0.001 \\
\hline E/R KD model & \\
\hline WU_APOPTOSIS_BY_CDKN1A_VIA_TP53 & 1 \\
KANNAN_TP53_TARGETS_UP $^{1}$ & 0.004 \\
TP53_DOWNSTREAM_TARGETS $^{2}$ & 0.023 \\
\hline
\end{tabular}

${ }^{1}$ GSEA analysis for "curated gene sets" (MSigDB: C2)

${ }^{2}$ GSEA analysis for "manually curated gene set"

b
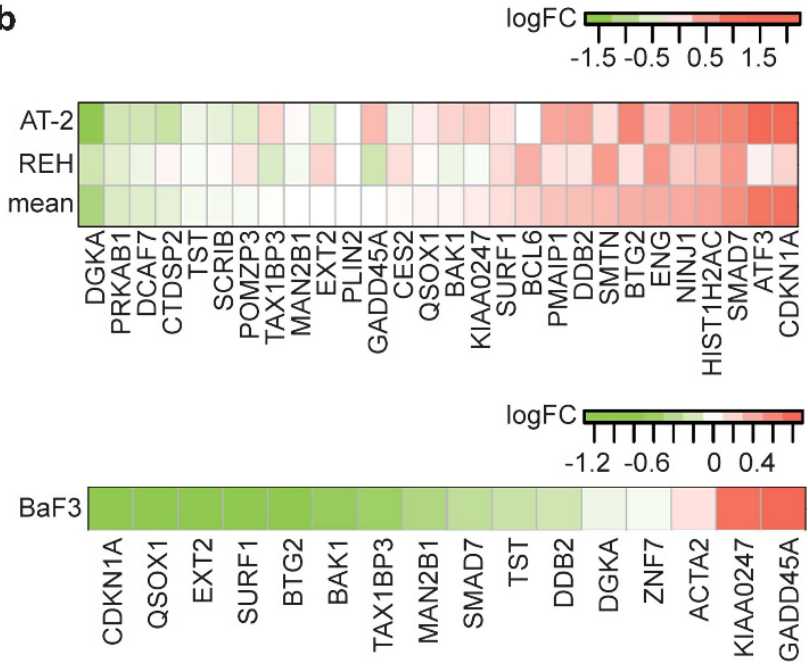

C
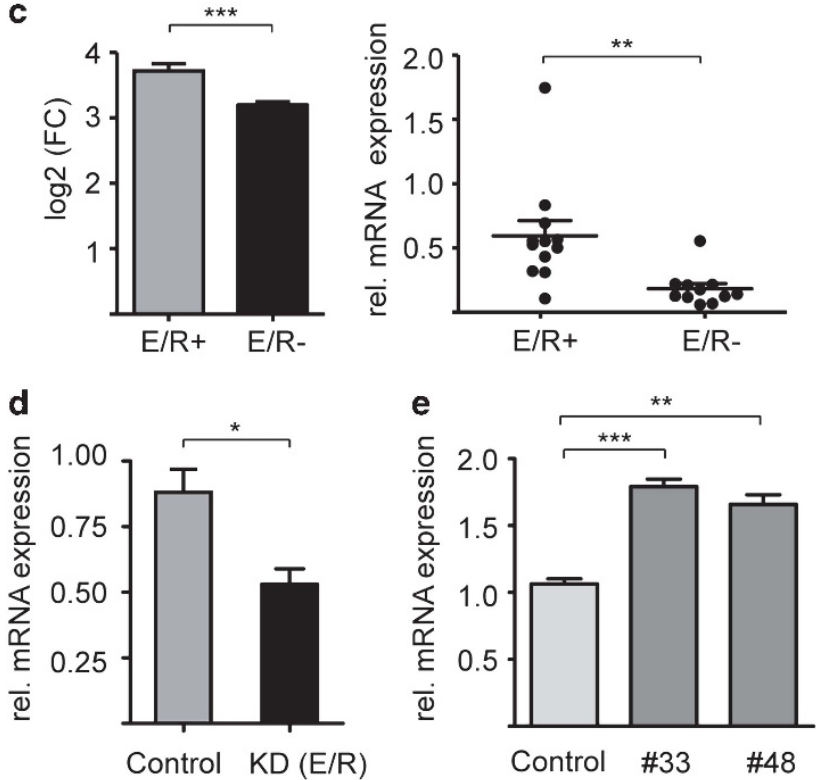

$E / R$

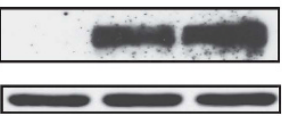


cells after $E / R$ KD (Figure $1 \mathrm{~d}$ ) and higher in E/R-expressing $\mathrm{Ba} / \mathrm{F} 3$ clones compared with parental cells (Figure 1e). These results strongly imply that $E / R$ specifically attenuates the $p 53$ pathway through upregulation of MDM2. Of note, none of the various leukemia expression profiles provided any evidence for a similar participation of $M D M X$, which is the second major negative regulator of $\mathrm{p} 53,{ }^{13}$ in E/R-positive cases.

MDM2 transcription is regulated by two promoters, which are activated in a p53-dependent and p53-independent manner, respectively. ${ }^{27}$ As in E/R-positive leukemias only the p53-dependent transcripts are differentially regulated, we investigated how E/R regulates the MDM2 P2 promoter as a function of p53 expression by performing luciferase reporter assays using the human isogenic $\mathrm{p} 53^{+/+}$and $\mathrm{p} 53^{-/-}$disparate HCT116 cell lines. ${ }^{28}$ We transiently transfected both of them with either an empty vector or various combinations and amounts of vector constructs that contained the $\mathrm{P} 2$ promoter sequences and the fusion transcript. E/R expression leads to an up to four-fold increase of MDM2 promoter activity (Figure 2a) and transcription (Supplementary Figure 1) in wt p53, but also, albeit to a lesser extent, in $\mathrm{p} 3^{-1-}$ cells (Figure 2a). These findings provide convincing evidence that E/R activates MDM2 transcription in an autonomous and dose-dependent manner.

Therefore, we presumed that MDM2 activation is most likely achieved through a direct promoter binding of the E/R protein. This notion is based on the fact that $E / R$ acts as an aberrant transcription factor via its RHD in a cell context-dependent manner and either represses or directly activates RUNX1 targets, as recently reported for the erythropoietin receptor. ${ }^{2,20,29-33}$ Moreover, there are also good indications that p53 is not the only direct regulator of the MDM2 P2 promoter. $^{13}$

To identify putative RUNX1-binding sites, we screened the MDM2 P2 promoter region in silico with Consite (http://asp.ii. uib.no:8090/cgi-bin/CONSITE/consite/), PATCH Search (http://www. gene-regulation.com/cgi-bin/pub/programs/patch/bin/patch.cgi) and JASPAR (http://jaspar.genereg.net/). All three programs identified one putative binding site (CCTGTGGGC) 184-bp upstream of the transcription start site in MDM2 exon 2 (Figure 2b). To check whether E/R binds to this site, we performed ChIP assays with HEK 293T cells, which stably express either E/R-V5, $\triangle$ RHD E/R-V5 or contain an empty vector-V5. Cells were fixed, sonicated and incubated with either the anti-V5 antibody or the negative control antibody (mlgG). After isolation and purification of the immunoprecipitated DNA, SYBR green quantitative PCR was performed using primers that were specific for the putative RUNX1-binding site. The E/R-harboring cells were enriched $\sim 5$-fold for the RUNX1 motif-positive MDM2 containing site proving that the chimeric protein occupies the respective chromatin area in vivo (Figure $2 \mathrm{c}$ ). The corresponding MDM2 expression is shown in the Supplementary Information (Supplementary Figure 2). These results provide first evidence that $E / R$ directly binds to and positively regulates MDM2 transcription from the P2 promoter.

Inhibition of MDM2 reactivates the p53 pathway

Under physiological conditions, MDM2 has a short half-life and is a crucial negative modulator of p53 transcription. Pre-existing genetic lesions that increase or stabilize MDM2 expression thus reduce p53 levels and consequently abrogate p53 signaling. ${ }^{13,14}$ As proteasomal degradation is not compromised in $E / R$-positive leukemic cell lines (data not shown), we tested whether Nutlin-3, a small molecule that disrupts the MDM2/p53 interaction, can reactivate $\mathrm{p} 53$ signaling. ${ }^{13}$

For this purpose, we exposed $E / R$-positive leukemic cell lines to various clinically meaningful concentrations $(1-10 \mu \mathrm{m})$ of Nutlin-3. This treatment led to a substantial accumulation of the p53 protein as well as the expression of its direct targets p21, MDM2 a

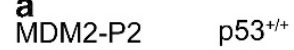
p5 $3^{-1}$
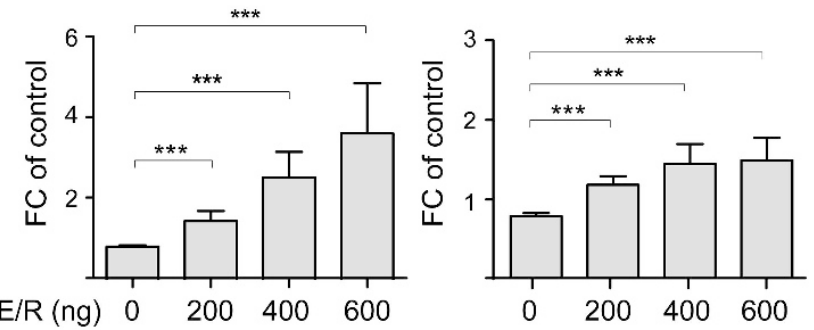

$E / R$

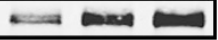

$\leftrightarrow$
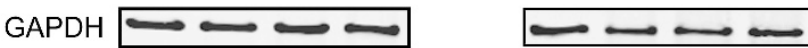

b

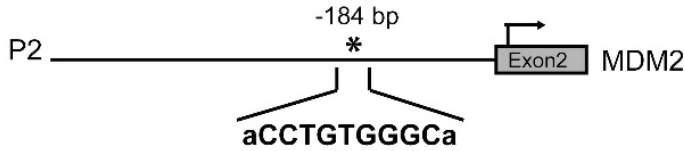

c

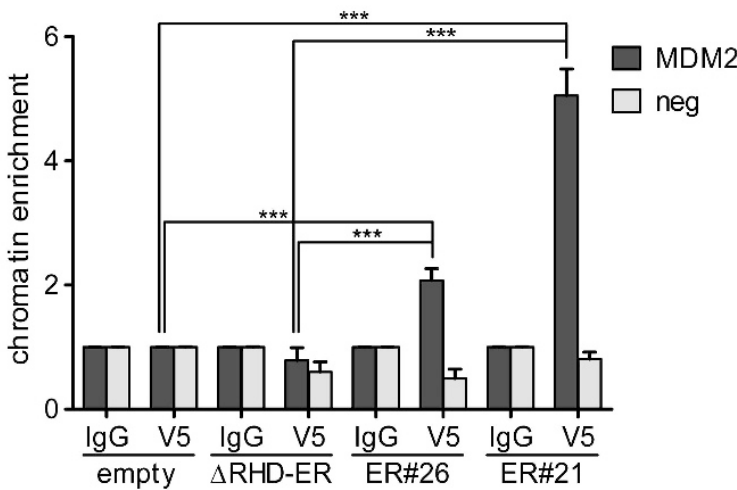

Figure 2. $E / R$ transactivates $M D M 2$ by binding to an endogenous RUNX1 motif. (a) Luciferase assays were performed using the MDM2 P2 promoter construct to transfect HCT116 p53 $3^{+/+}$and $\mathrm{p} 53^{-1-}$ cells together with increasing amounts of E/R expression vector (indicated at the $x$ axis). Cells were co-transfected with core binding factor $\beta$ to enhance binding and TK Renilla as transfection control. The total amount of DNA for each experiment was kept constant. Luciferase activity of pooled data of three independent experiments is depicted as fold-change (FC) of promoter activity of the empty vector. Welch's $t$-test, ${ }^{* * *} P \leqslant 0.001$. E/R expression by western blot analysis of the respective samples is shown at the bottom of the figure. (b) In silico search for putative RUNX1-binding sites in the MDM2 P2 promoter (895 bp) reporter construct used in (a) revealed one RUNX-binding motif 184-bp upstream of the transcription start (indicated by an asterisk). (c) ChIP assays at this putative RUNX1binding site in HEK 293T cells stably expressing E/R (clones ER\#21 and ER\#26) and various controls ( $\triangle R H D-E R$; empty vector). Anti-V5 antibody was used for immunoprecipitation of $E / R$ and mouse IgG as isotype control. MDM2 indicates the specific binding of E/R to the genomic region and 'neg' is an MDM2 genomic region negative for E/R-binding sites. Welch's $t$-test, ${ }^{* * *} P \leqslant 0.001$. ER\#26 vs $\triangle$ RHD-ER and empty vector are all $P \leqslant 0.001$.

and the pro-apoptotic BAX and PUMA proteins in all instances (Figure $3 a$ ). As the consequences of p53 reactivation depend on the specific cellular context, ${ }^{34,35}$ we investigated how Nutlin-3 affects the cell cycle distribution as well as the viability of cells and their propensity to undergo apoptosis. As shown in Figures 3b-e, Nutlin-3 treatment significantly reduced viability and increased apoptosis in a dose-dependent manner. Consistent with the induction of p21 it also raised the proportion of cells in the G0/G1 and lowered that of cells in the S-phase (Figure 3f, Supplementary Figure 3). Collectively, these data demonstrate that Nutlin-3 
a
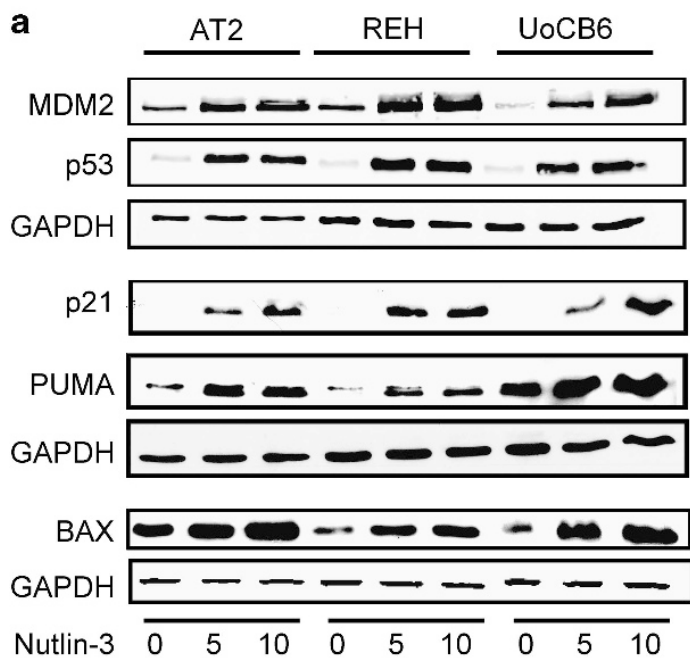
$(\mu \mathrm{M})$

d Nutlin-3
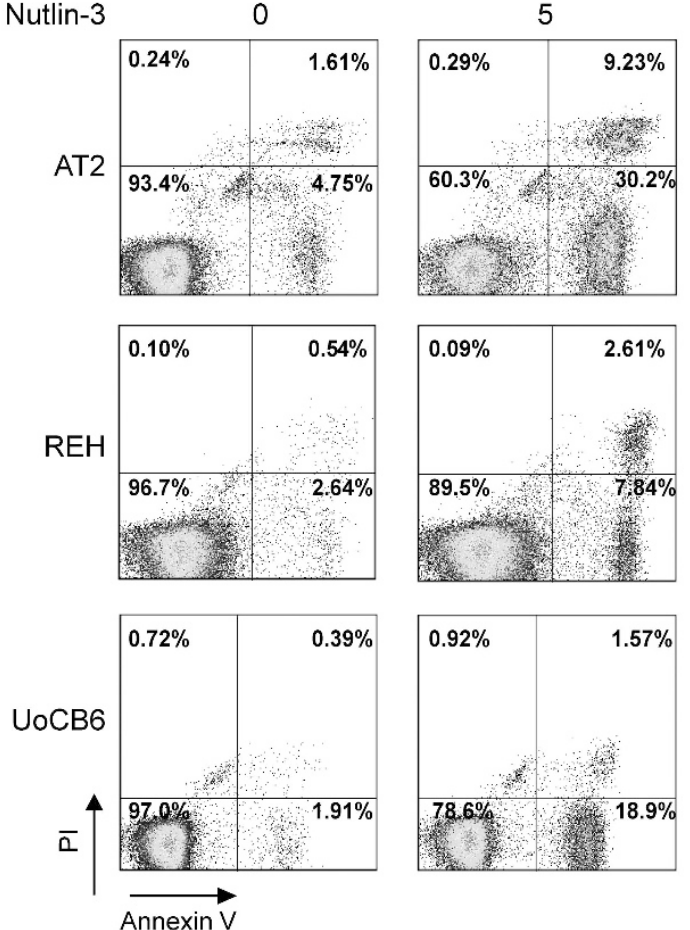

Annexin V

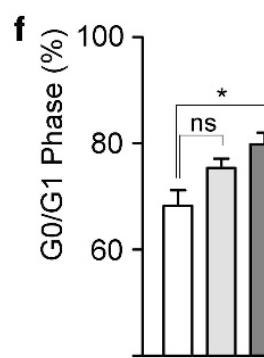

AT2

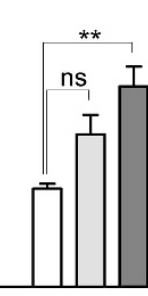

$\mathrm{REH}$

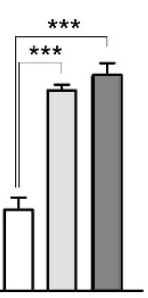

UoCB6

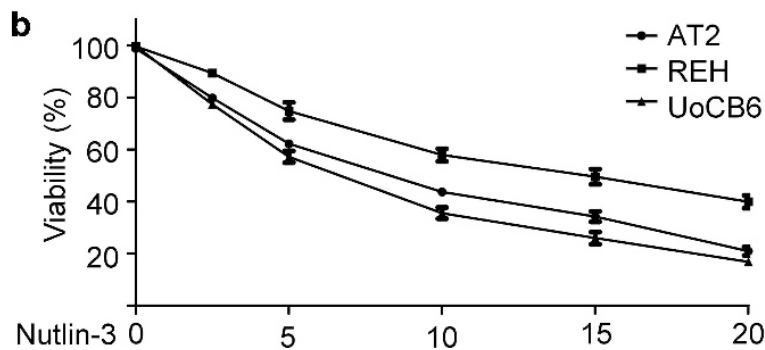

$(\mu \mathrm{M})$

c

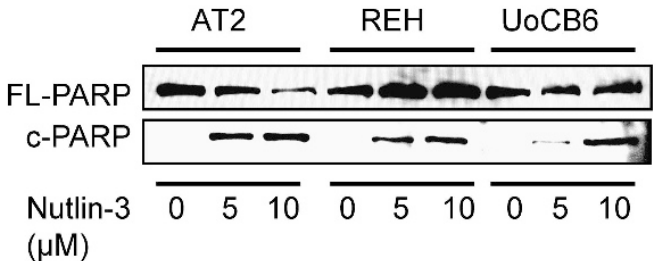

e
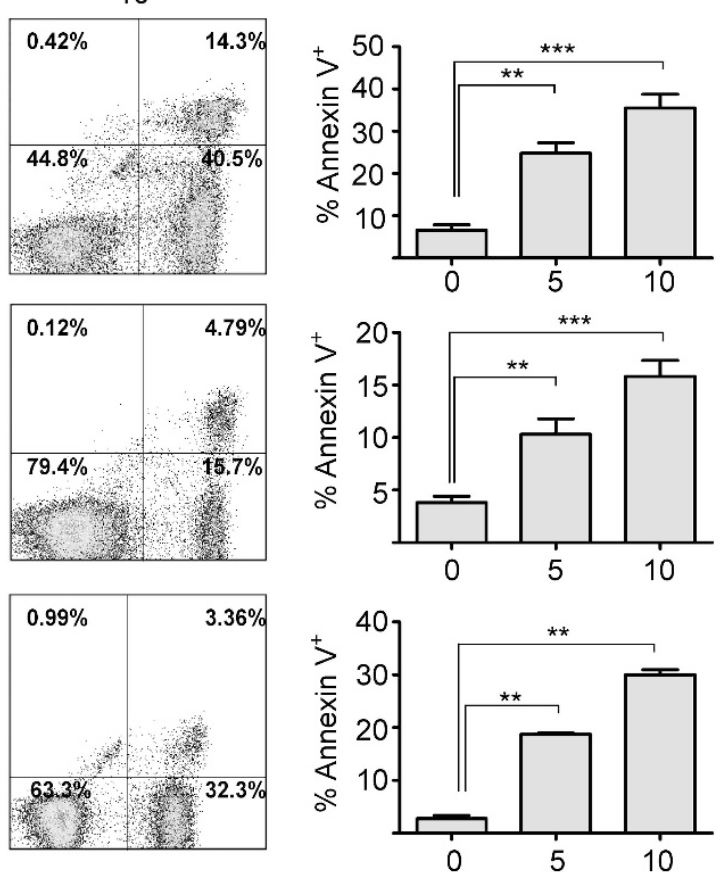

Figure 3. Reactivation of $\mathrm{p} 53$ by Nutlin-3 in E/R-expressing leukemic cell lines. (a) Western blot analysis of AT2, REH and UoCB6 cells confirms accumulation of p53 and induction of downstream targets MDM2, PUMA, BAX and p21 upon exposure to Nutlin-3. Glyceraldehyde 3-phosphate dehydrogenase (GAPDH) was used as loading control. Results are representative of three independent experiments. (b) Viability (3-(4,5-dimethylthiazol-2-yl)-2,5-diphenyltetrazoliumbromid) assays of cell lines upon exposure to Nutlin-3. Pooled data of three independent experiments are shown. (c-e) Apoptosis was assessed by western blot analysis for poly ADP-ribose polymerase cleavage (FL, full-length; c, cleaved) (shown in c), annexin-V/propidium iodide (PI) staining (representative histograms in d) and proportions of Annexin-V/PI + cells (\%) (e) of three independent experiments. (f) Distribution of cells in G0/G1 and S phase relative to carrier control upon exposure to Nutlin-3. Data are derived from at least three independent experiments. Welch's $t$-test, ${ }^{*} P \leqslant 0.05,{ }^{* *} P \leqslant 0.01,{ }^{* * *} P \leqslant 0.001$. NS, not significant. 
restores p53 function in $E / R$-expressing leukemic cell lines and underscore the relevance of p53 pathway signaling alterations for the disease.

Nutlin-3 enhances drug-induced apoptosis in E/R-positive primary leukemias

We also checked whether the addition of Nutlin-3 can potentiate the apoptosis-inducing effect of DNA-damaging and p53-activating chemotherapeutic drugs, such as daunorubicin, asparaginase and vincristine, which are currently used worldwide in all major childhood ALL treatment protocols. ${ }^{1,26}$ The first two drugs activate the DNA damage checkpoint mainly by inducing DNA doubleand/or single-strand breaks, whereas the latter by disrupting the mitotic spindle.

We exposed the E/R-positive REH and UoCB6 cell lines to low concentrations of Nutlin-3 (2.5 and $5 \mu \mathrm{m})$ together with the aforementioned drugs using established concentrations (daunorubicin, 0.01 and $0.05 \mu \mathrm{g} / \mathrm{ml}$; asparaginase, 0.05 and $0.5 \mathrm{IU} / \mathrm{ml}$; vincristine, 0.5 and $1 \mathrm{ng} / \mathrm{ml}){ }^{36-38} 3$-(4,5-Dimethylthiazol-2-yl)-2,5diphenyltetrazoliumbromid assays were performed $48 \mathrm{~h}$ after co-exposure with the chemotherapeutic agents and revealed a reduction of cell survival by up to $80 \%$ and increase of apoptosis by $42 \%$ (for daunorubicin and asparaginase) and 64\% (for vincristine), whereas the comparable rates with Nutlin-3 alone were significantly lower (Figure 4 and Supplementary Figure 4).

To confirm that Nutlin-3 exerts a similar effect also on primary leukemias, we used E/R-positive samples with $>90 \%$ blasts from children who were consecutively enrolled in the Austrian ALL-Berlin-Frankfurt-Münster 2000 protocol. Appropriate E/Rnegative samples were used as control. As an initial assessment by western blot and flow cytometry yielded equal estimates of p53 protein levels (Figure 5a), we used only flow cytometry for the consecutive analyses. As observed in the cell lines, Nutlin-3 exposure increased p53 protein levels and the proportion of apoptotic cells in a dose-dependent manner in all samples (Figure 5b). In accordance with the generally higher MDM2 levels, however, also the apoptosis rates were significantly more pronounced in $E / R$-positive than in $E / R$-negative samples (Supplementary Figure 5).

\section{DISCUSSION}

The $E / R$ fusion gene drives not only the leukemic transformation process but its sustained expression is also indispensable for the progression and maintenance of the disease. ${ }^{11,39}$ Previous evidence has already indicated that suppression of p53 signaling is a crucial element in this development and that the chimeric E/R protein does probably directly deregulate this pathway, although the mode of its action has remained unexplored so far. ${ }^{12}$ Herein we now show, in various cellular model systems and primary leukemias, that the aberrant $E / R$ transcription factor binds to the MDM2 P2 promoter and consequently upregulates MDM2 in a direct and p53-independent manner. Nutlin-3, a specific inhibitor of the MDM2/p53 interaction, is able to relieve the ensuing p53 attenuation, which leads to cell cycle arrest and enhanced apoptosis in the affected cells.

The p53 signaling pathway is probably affected in the vast majority of all types of cancer in one way or the other. Its function can be disrupted either by various types of genetic alterations (as for instance, TP53 mutations, CDKN2A deletions) or by a sustained expression of $M D M 2$, the main negative $\mathrm{p} 53$ regulator. This latter mode of inactivation is also observed in $E / R$-positive leukemias. So far, the focus of interest in MDM2 overexpression has been on drug-resistant childhood ALL cases, whereas its potential relevance in $E / R$-positive cases has been largely disregarded because of the comparably favorable outcome of these leukemias.
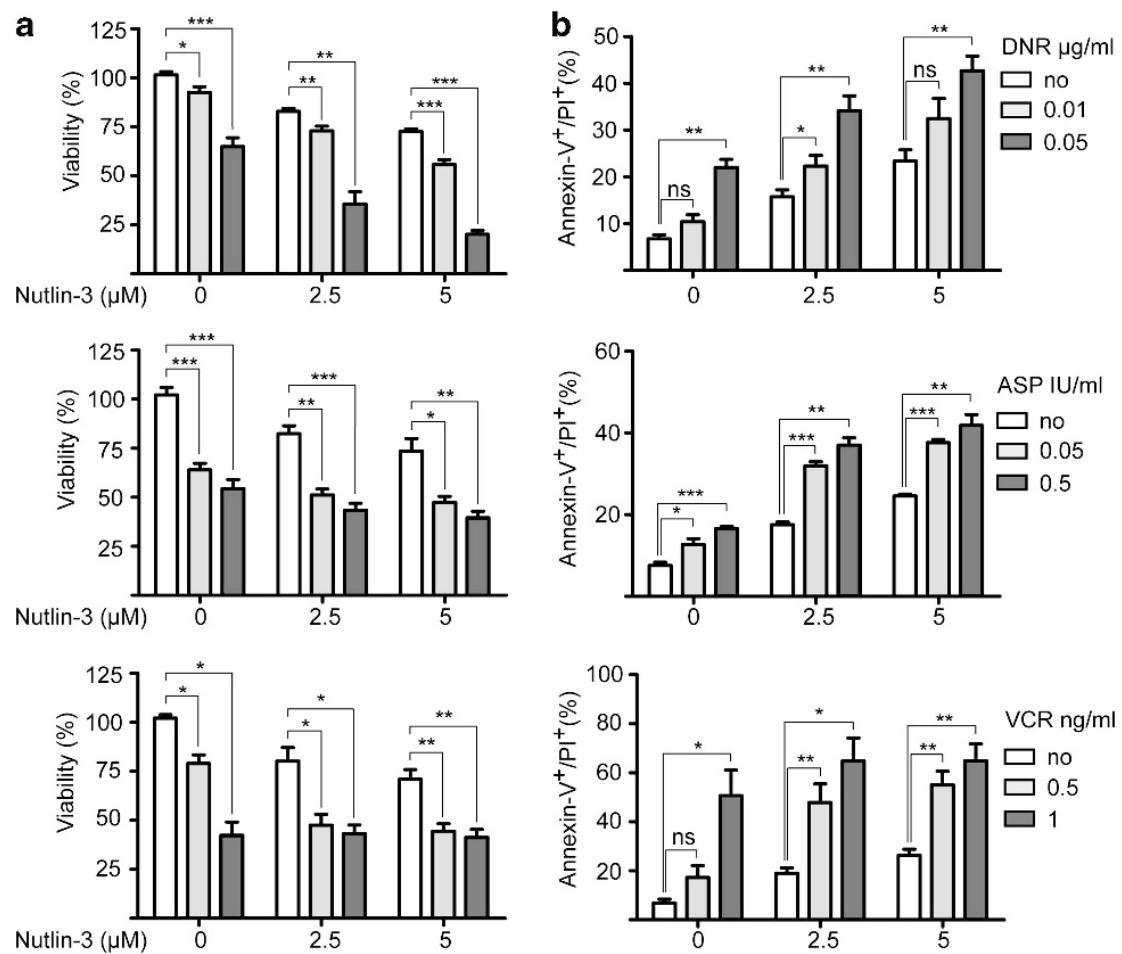

Figure 4. Nutlin-3 enhances effects of chemotherapeutic agents in E/R-expressing cell lines. REH cells were exposed to daunorubicin (DNR), vincristine (VCR) and asparaginase (ASP) alone and in combination with Nutlin-3. (a) Viability was determined by 3-(4,5-dimethylthiazol-2-yl)2,5-diphenyltetrazoliumbromid assay and is indicated relative to carrier control. (b) Apoptotic fractions were measured by flow cytometry. The percentage of apoptotic cells (annexinV $+/$ propidium iodide $(\mathrm{PI})+$ ) is shown. Data were derived from at least three independent experiments. Welch's $t$-test, ${ }^{*} P \leqslant 0.05,{ }^{* *} P \leqslant 0.01,{ }^{* * *} P \leqslant 0.001$. NS, not significant. 
a
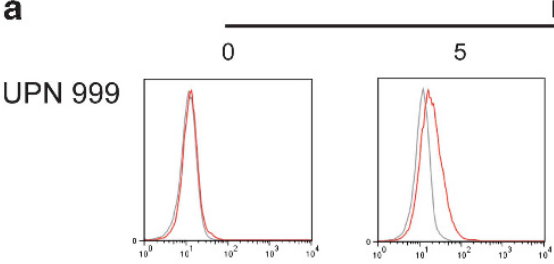

Nutlin-3
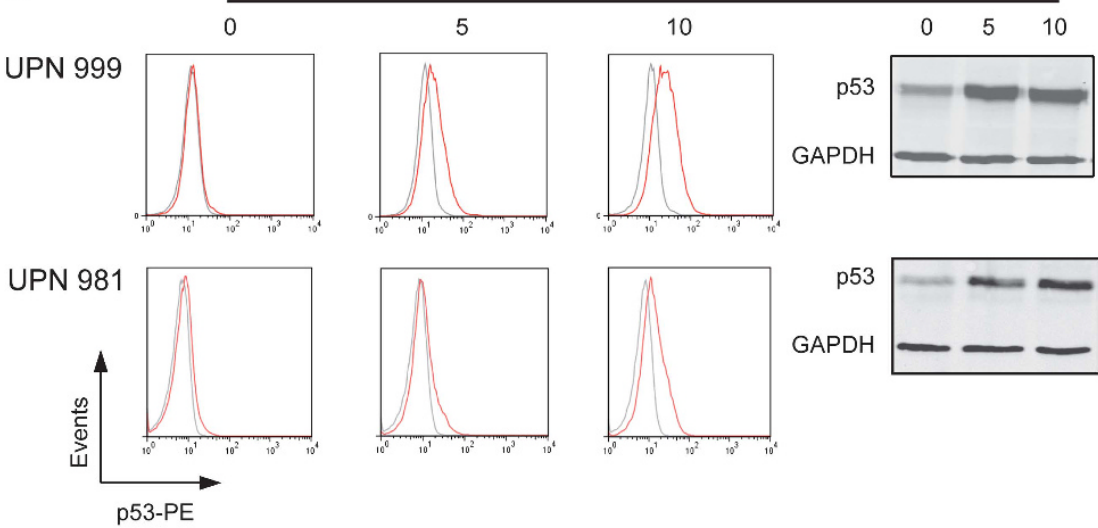

b

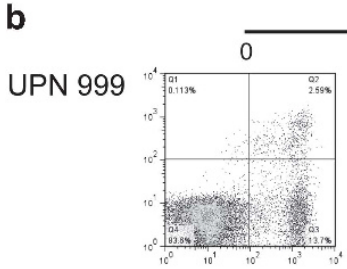

Nutlin-3
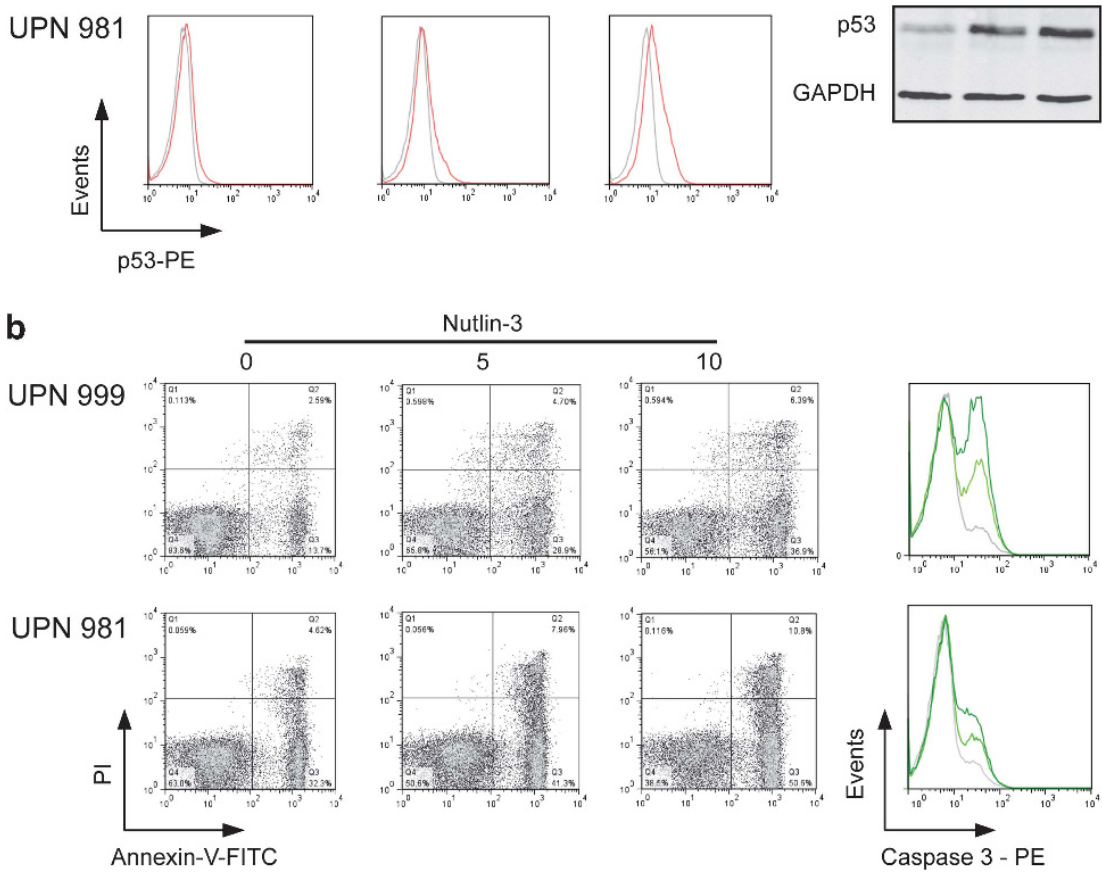

Figure 5. Nutlin-3 reactivates $\mathrm{p} 53$ and induces apoptosis in primary ALL samples. Representative examples of $E / R$-positive (UPN 999 , top) and E/R-negative (UPN 981, bottom) primary leukemia samples upon exposure to Nutlin-3 are depicted. (a) Left part: flow cytometric analysis of p53 expression (red) and respective isotype control (gray); corresponding western blot using glyceraldehyde 3-phosphate dehydrogenase (GAPDH) as control (right). (b) Apoptosis was assessed by AnnexinV/propidium iodide (PI; left) and cleaved caspase 3 stainings (right); green lines, upon exposure to Nutlin-3 (light green, $5 \mu \mathrm{m}$; dark green, $10 \mu \mathrm{m}$ concentration); gray lines, untreated control. PE, phycoerythrin.

However, MDM2 overexpression seems to be a distinct and rather unique feature of $E / R$-positive $A L L$, because to this extent it is only found in this particular genetic subgroup. Our earlier functional analyses of leukemic cell lines, in which the $E / R$ fusion gene had been knocked down, already implied that MDM2 overexpression is the essential factor that abolishes p53 function. ${ }^{12}$ Moreover, no other possible activation routes, such as MDM2 gene amplifications, are encountered in such cases. ${ }^{4,10,40}$ The only other conceivable route that might perhaps also lead to MDM2 upregulation and consequently to the impairment of p53 signaling is the loss of p14 ${ }^{\mathrm{ARF}}$, a CDKN2A-encoded inhibitor of MDM2, which is deleted in up to $30 \%$ of $E / R$-positive cases. ${ }^{4,10,41}$

Our observation that $\mathrm{E} / \mathrm{R}$ activates MDM2 directly via binding to a RUNX1 motif in the P2 promoter region fits perfectly to its role as aberrant transcription factor. It adds evidence that $E / R$ can regulate the respective RUNX1 target genes not only by repressing but also by activating them. ${ }^{29}$ This aspect was first noted by Neil's group and was based on data that were obtained in a murine fibroblast cell line. ${ }^{31}$ More recently, it was further corroborated in human $E / R$-positive cell lines and primary leukemic samples by demonstrating that $E / R$ binds and activates the promoter of the erythropoietin receptor, whose atypical expression seems to be another characteristic feature of $E / R$-positive leukemias. ${ }^{31-33}$ On a broader and more general scale, the significance of this phenomenon is also supported by the results of our previous fusion gene KD experiment, in which it became evident that approximately half of all E/R-regulated genes are upregulated, a substantial number of which contain putative RUNX1-binding sites in their promoter regions. ${ }^{12}$ These RUNX1-binding motifs were verified by ChIP sequencing in material that derived from human hematopoietic stem and progenitor cells as well as megakaryocytes. ${ }^{42,43}$

To study the effects of an impeded MDM2 function on p53, we exposed the respective cells to Nutlin-3, a small molecule inhibitor of the MDM2/p53 interaction. On the basis of its ability to reactivate p53 in malignancies with a wt TP53 and because toxicity of this agent is negligible in normal cells, it is already being tested in clinical trials. ${ }^{13,44}$ Previous studies have found that Nutlin-3 variably arrests the cell cycle and induces apoptosis in leukemic cell lines with a wt TP53, the extent of which depends on the level of MDM2 expression. ${ }^{13}$ In our experiments, however, Nutlin-3induced apoptosis and merely a G0/G1 arrest but not, as reported previously in some cancers, also a $\mathrm{G} 2 / \mathrm{M}$ arrest. ${ }^{13}$ Although we cannot rule out that cells arrested in G2/M did not accumulate and appear as a discernible fraction because they immediately underwent apoptosis, it seems more likely that an E/R-mediated repression of $M A D 2 L 1$, together with the abrogation of the mitotic cell cycle control, interfered with the potential p53-mediated G2/M check point function. ${ }^{20}$

The E/R-induced upregulation of MDM2 in $\mathrm{Ba} / \mathrm{F} 3$ murine pro- $\mathrm{B}$ cells indicates that this occurs merely as a function of the fusion gene early on during leukemia development and is found in the absence of secondary aberrations. In support of this view, the expression of RUNX1-ETO, a related RUNX1 gene fusion that occurs 


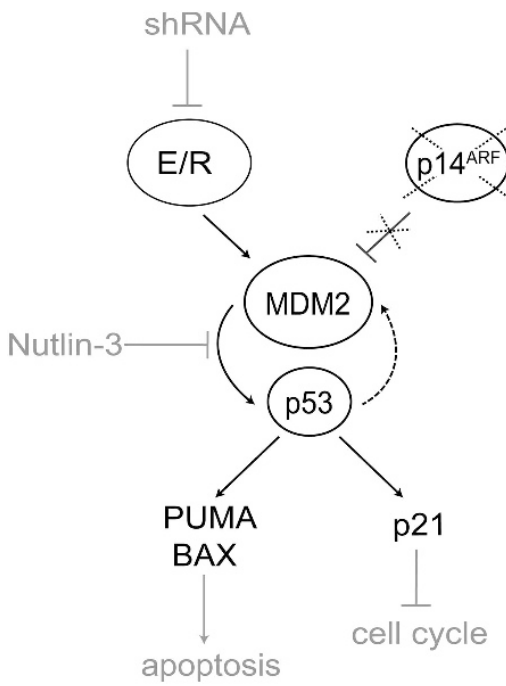

Figure 6. Model for $p 53$ pathway repression by E/R. E/R inhibits $p 53$ by upregulating MDM2 expression, a process that can be reversed by suppressing E/R with short hairpin RNA. Coexisting deletions of CDKN2A, which encodes the MDM2 inhibitor p14 ${ }^{\mathrm{ARF}}$, can further consolidate MDM2 expression and the ensuing effects on p53. Disrupting this inappropriate MDM2/p53 interaction with the small molecule inhibitor Nutlin-3 restores p53 function leading to upregulation of p21, BAX and PUMA and consequently arrests the cell cycle and induces apoptosis in E/R-positive cells.

in acute myeloid leukemias, also upregulates MDM2 and thereby represses p53 signaling in hematopoietic stem cells. ${ }^{45}$ Moreover, treatment of these transduced cells with Nutlin-3 reversed the observed increased proliferation and self-renewal. Similarly, Nutlin-3 reduced proliferation in E/R-positive leukemic cells by cell cycle arrest and apoptosis, which was enhanced upon exposure to chemotherapeutic drugs. These effects were more pronounced in primary $E / R$-positive than $E / R$-negative cases, which again underlines that this phenomenon is a unique and inherent feature of this particular genetic subgroup. In this context, it is also of special interest that the activation of the phosphatidylinositol 3 kinase/Akt pathway promotes the phosphorylation of MDM2 and its localization to the nucleus where it binds to p53 and enhances ubiquitin-mediated degradation. ${ }^{44,46}$ Of note, activation of the phosphatidylinositol 3 kinase/Akt pathway emerged also as one of the prominent features in our E/R fusion gene KD experiments. ${ }^{11}$

On the basis of our results, we present a model that outlines how E/R impairs p53 function and promotes leukemia development and progression (Figure 6). In this scenario, E/R directly upregulates MDM2 and thereby represses p53, which is a prerequisite for the competitive survival advantage of the transformed cells. This unstable genomic state might also predispose the cells to the development of additional genetic lesions, such as CDKN2A deletions. The ensuing reduction of the encoded $\mathrm{p} 14^{\mathrm{ARF}}$ protein finally mitigates the $\mathrm{p} 14^{\mathrm{ARF}}$-mediated control of MDM2 activity and thereby further enhances the degradation of $\mathrm{p} 53$

In conclusion, we have provided strong evidence that $E / R$ abrogates p53 signaling through the direct induction of MDM2 expression, a cellular attribute that can be reversed in vitro by the inhibition of MDM2. Taken together, these finding suggest (1) that disruption of the p53 signaling pathway is an important mechanism, which promotes cell survival and probably also facilitates the acquisition of secondary aberrations and (2) that the pharmacological inhibition of the respective MDM2/p53 interaction with small molecules may provide new specific avenues for individualized therapeutic interventions in this group of patients.

\section{CONFLICT OF INTEREST}

The authors declare no conflict of interest.

\section{ACKNOWLEDGEMENTS}

This work was supported in part by the Austrian Science Fund (FWF): [P 22073-B19].

\section{AUTHOR CONTRIBUTIONS}

UK and MM designed and performed experiments, interpreted data and drafted the manuscript. CP, AM and MZ performed experiments. AA contributed patient samples. MK performed bioinformatics analyses. OAH interpreted data and wrote the paper. RP-G conceived and supervised the study, interpreted data and wrote the manuscript. All authors read and agreed to the final version of the manuscript.

\section{REFERENCES}

1 Pui CH, Carroll WL, Meshinchi S, Arceci RJ. Biology, risk stratification, and therapy of pediatric acute leukemias: an update. J Clin Oncol 2011; 29: 551-565.

2 Zelent A, Greaves M, Enver T. Role of the TEL-AML1 fusion gene in the molecular pathogenesis of childhood acute lymphoblastic leukaemia. Oncogene 2004; 23: 4275-4283.

3 Conter V, Bartram CR, Valsecchi MG, Schrauder A, Panzer-Grumayer R, Moricke A et al. Molecular response to treatment redefines all prognostic factors in children and adolescents with B-cell precursor acute lymphoblastic leukemia: results in 3184 patients of the AIEOP-BFM ALL 2000 study. Blood 2010; 115: 3206-3214.

4 Kuster L, Grausenburger R, Fuka G, Kaindl U, Krapf G, Inthal A et al. ETV6/RUNX1-positive relapses evolve from an ancestral clone and frequently acquire deletions of genes implicated in glucocorticoid signaling. Blood 2011; 117: $2658-2667$

5 Malempati S, Gaynon PS, Sather H, La MK, Stork LC. Outcome after relapse among children with standard-risk acute lymphoblastic leukemia: Children's Oncology Group study CCG-1952. J Clin Oncol 2007; 25: 5800-5807.

6 Diakos C, Krapf G, Gerner C, Inthal A, Lemberger C, Ban J et al. RNAi-mediated silencing of TEL/AML1 reveals a heat-shock protein- and survivin-dependent mechanism for survival. Blood 2007; 109: 2607-2610.

7 Diakos C, Zhong S, Xiao Y, Zhou M, Vasconcelos GM, Krapf G et al. TEL-AML1 regulation of survivin and apoptosis via miRNA-494 and miRNA-320a. Blood 2010; 116: 4885-4893.

8 Schindler JW, Van Buren D, Foudi A, Krejci O, Qin J, Orkin SH et al. TEL-AML1 corrupts hematopoietic stem cells to persist in the bone marrow and initiate leukemia. Cell Stem Cell 2009; 5: 43-53.

9 Hong D, Gupta R, Ancliff P, Atzberger A, Brown J, Soneji S et al. Initiating and cancer-propagating cells in TEL-AML1-associated childhood leukemia. Science 2008; 319: 336-339.

10 Mullighan CG, Goorha S, Radtke I, Miller CB, Coustan-Smith E, Dalton JD et al. Genome-wide analysis of genetic alterations in acute lymphoblastic leukaemia. Nature 2007; 446: 758-764.

11 Fuka G, Kantner HP, Grausenburger R, Inthal A, Bauer E, Krapf G et al. Silencing of ETV6/RUNX1 abrogates PI3K/AKT/mTOR signaling and impairs reconstitution of leukemia in xenografts. Leukemia 2012; 26: 927-933.

12 Fuka G, Kauer M, Kofler R, Haas OA, Panzer-Grumayer R. The leukemia-specific fusion gene ETV6/RUNX1 perturbs distinct key biological functions primarily by gene repression. PLoS One 2011; 6: e26348.

13 Wade M, Li YC, Wahl GM. MDM2, MDMX and p53 in oncogenesis and cancer therapy. Nat Rev Cancer 2013; 13: 83-96.

14 Brown CJ, Lain S, Verma CS, Fersht AR, Lane DP. Awakening guardian angels: drugging the p53 pathway. Nat Rev Cancer 2009; 9: 862-873.

15 Cheok CF, Verma CS, Baselga J, Lane DP. Translating p53 into the clinic. Nat Rev Clin Oncol 2011; 8: 25-37.

16 Hof J, Krentz S, van Schewick C, Korner G, Shalapour S, Rhein P et al. Mutations and deletions of the TP53 gene predict nonresponse to treatment and poor outcome in first relapse of childhood acute lymphoblastic leukemia. J Clin Oncol 2011; 29: 3185-3193.

17 Zhang J, Mullighan CG, Harvey RC, Wu G, Chen X, Edmonson M et al. Key pathways are frequently mutated in high-risk childhood acute lymphoblastic leukemia: a report from the Children's Oncology Group. Blood 2011; 118: 3080-3087.

18 Fears S, Vignon C, Bohlander SK, Smith S, Rowley JD, Nucifora G. Correlation between the ETV6/CBFA2 (TEL/AML1) fusion gene and karyotypic abnormalities in children with B-cell precursor acute lymphoblastic leukemia. Genes Chromosomes Cancer 1996; 17: 127-135. 
19 Kim DH, Moldwin RL, Vignon C, Bohlander SK, Suto Y, Giordano L et al. TEL-AML1 translocations with TEL and CDKN2 inactivation in acute lymphoblastic leukemia cell lines. Blood 1996; 88: 785-794.

20 Krapf G, Kaindl U, Kilbey A, Fuka G, Inthal A, Joas R et al. ETV6/RUNX1 abrogates mitotic checkpoint function and targets its key player MAD2L1. Oncogene 2010; 29: 3307-3312.

21 Phelps M, Darley M, Primrose JN, Blaydes JP. p53-independent activation of the hdm2-P2 promoter through multiple transcription factor response elements results in elevated hdm2 expression in estrogen receptor alpha-positive breast cancer cells. Cancer Res 2003; 63: 2616-2623.

22 Gentleman RC, Carey VJ, Bates DM, Bolstad B, Dettling M, Dudoit S et al. Bioconductor: open software development for computational biology and bioinformatics. Genome Biol 2004; 5: R80.

23 Den Boer ML, van Slegtenhorst M, De Menezes RX, Cheok MH, Buijs-Gladdines JG, Peters ST et al. A subtype of childhood acute lymphoblastic leukaemia with poor treatment outcome: a genome-wide classification study. Lancet Oncol 2009; 10 $125-134$

24 Wu Z, Irizarry RA, Gentleman R, Martinez-Murillo F, Spencer F. A model-based background adjustment for oligonucleotide expression arrays. J Am Stat Assoc 2004; 99: 909-917.

25 Wettenhall JM, Smyth GK. LimmaGUI: a graphical user interface for linear modeling of microarray data. Bioinformatics 2004; 20: 3705-3706.

26 Riley $\mathrm{T}$, Sontag $\mathrm{E}_{c}$ Chen $\mathrm{P}$, Levine A. Transcriptional control of human p53-regulated genes. Nat Rev Mol Cell Biol 2008; 9: 402-412.

27 Bartel F, Taubert H, Harris LC. Alternative and aberrant splicing of MDM2 mRNA in human cancer. Cancer Cell 2002; 2: 9-15.

28 Bunz F, Dutriaux A, Lengauer C, Waldman T, Zhou S, Brown JP et al. Requirement for p53 and p21 to sustain G2 arrest after DNA damage. Science 1998; 282: 1497-1501.

29 Hiebert SW, Sun W, Davis JN, Golub T, Shurtleff S, Buijs A et al. The $t(12 ; 21)$ translocation converts AML-1B from an activator to a repressor of transcription. Mol Cell Biol 1996; 16: 1349-1355

30 Morrow M, Samanta A, Kioussis D, Brady HJ, Williams O. TEL-AML1 preleukemic activity requires the DNA binding domain of AML1 and the dimerization and corepressor binding domains of TEL. Oncogene 2007; 26: 4404-4414.

31 Wotton S, Terry A, Kilbey A, Jenkins A, Herzyk P, Cameron E et al. Gene array analysis reveals a common Runx transcriptional programme controlling cell adhesion and survival. Oncogene 2008; 27: 5856-5866.

32 Inthal A, Krapf G, Beck D, Joas R, Kauer MO, Orel L et al. Role of the erythropoietin receptor in ETV6/RUNX1-positive acute lymphoblastic leukemia. Clin Cancer Res 2008; 14: 7196-7204

33 Torrano V, Procter J, Cardus P, Greaves M, Ford A. ETV6-RUNX1 promotes survival of early $B$ lineage progenitor cells via a dysregulated erythropoietin receptor. Blood 2011; 118: 4910-4918.

34 Xu-Monette ZY, Medeiros L, Li Y, Orlowski RZ, Andreeff M, Bueso-Ramos CE et al. Dysfunction of the TP53 tumor suppressor gene in lymphoid malignancies. Blood 2012; 119: 3668-3683.
35 Pant V, Quintas-Cardama A, Lozano G. The p53 pathway in hematopoiesis: lessons from mouse models, implications for humans. Blood 2012; 120: 5118-5127.

36 Ramakers-van Woerden NL, Pieters R, Loonen AH, Hubeek I, van Drunen E, Beverloo HB et al. TEL/AML1 gene fusion is related to in vitro drug sensitivity for L- asparaginase in childhood acute lymphoblastic leukemia. Blood 2000; 96: 1094-1099.

37 Naderi EH, Findley HW, Ruud E, Blomhoff HK, Naderi S. Activation of cAMP signaling inhibits DNA damage-induced apoptosis in BCP-ALL cells through abrogation of p53 accumulation. Blood 2009; 114: 608-618.

38 Frost BM, Forestier E, Gustafsson G, Nygren P, Hellebostad M, Jonsson OG et al. Translocation $\mathrm{t}(12 ; 21)$ is related to in vitro cellular drug sensitivity to doxorubicin and etoposide in childhood acute lymphoblastic leukemia. Blood 2004; 104 2452-2457.

39 Pui CH, Relling MV, Downing JR. Acute lymphoblastic leukemia. N Engl J Med 2004; 350: 1535-1548.

40 van Delft FW, Horsley S, Colman S, Anderson K, Bateman C, Kempski H et al. Clonal origins of relapse in ETV6-RUNX1 acute lymphoblastic leukemia. Blood 2011; 117: 6247-6254

41 Lilljebjorn H, Soneson C, Andersson A, Heldrup J, Behrendtz M, Kawamata N et al. The correlation pattern of acquired copy number changes in 164 ETV6/RUNX1positive childhood acute lymphoblastic leukemias. Hum Mol Genet 2010; 19: 3150-3158.

42 Wilson NK, Foster SD, Wang X, Knezevic K, Schutte J, Kaimakis $\mathrm{P}$ et al. Combinatorial transcriptional control in blood stem/progenitor cells: genome-wide analysis of ten major transcriptional regulators. Cell Stem Cell 2010; 7: 532-544.

43 Tijssen MR, Cvejic A, Joshi A, Hannah RL, Ferreira R, Forrai A et al. Genome-wide analysis of simultaneous GATA1/2, RUNX1, FLI1, and SCL binding in megakaryocytes identifies hematopoietic regulators. Dev Cell 2011; 20: 597-609.

$44 \mathrm{Gu} \mathrm{L}$, Zhu N, Findley HW, Zhou M. MDM2 antagonist nutlin-3 is a potent inducer of apoptosis in pediatric acute lymphoblastic leukemia cells with wild-type p53 and overexpression of MDM2. Leukemia 2008; 22: 730-739.

45 Abdul-Nabi AM, Yassin ER, Varghese N, Deshmukh H, Yaseen NR. In vitro transformation of primary human $\mathrm{CD} 34+$ cells by $\mathrm{AML}$ fusion oncogenes: early gene expression profiling reveals possible drug target in AML. PLoS One 2010; 5: e12464

46 Kojima K, Shimanuki M, Shikami M, Samudio IJ, Ruvolo V, Corn P et al. The dual PI3 kinase/mTOR inhibitor $\mathrm{Pl}-103$ prevents $\mathrm{p} 53$ induction by $\mathrm{Mdm} 2$ inhibition but enhances p53-mediated mitochondrial apoptosis in p53 wild-type AML. Leukemia 2008; 22: 1728-1736.

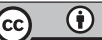

This work is licensed under a Creative Commons Attribution 3.0 Unported License. To view a copy of this license, visit http:// creativecommons.org/licenses/by/3.0/

Supplementary Information accompanies this paper on the Leukemia website (http://www.nature.com/leu) 